\title{
STABLE SAMPLING AND FOURIER MULTIPLIERS
}

\author{
BASARAB MATEI, YVES MEYER AND JOAQUIM ORTEGA-CERDÀ
}

\begin{abstract}
We study the relationship between stable sampling sequences for bandlimited functions in $L^{p}\left(\mathbb{R}^{n}\right)$ and the Fourier multipliers in $L^{p}$. In the case that the sequence is a lattice and the spectrum is a fundamental domain for the lattice the connection is complete. In the case of irregular sequences there is still a partial relationship.
\end{abstract}

\section{INTRODUCTION}

When $\omega>0,1<p<\infty$, and $f \in L^{p}(\mathbb{R})$ we write $f \in E_{\omega}^{p}$ if the Fourier transform $\hat{f}$ of $f$ vanishes outside $[-\omega, \omega]$. If $0<h \leq \pi / \omega$ the Shannon theorem states that any $f \in E_{\omega}^{p}$, can be completely recovered from its samples $f(k h), k \in \mathbb{Z}$. When $h=\pi / \omega$, the map $S_{\omega}: f \mapsto$ $\sqrt{h} f(k h), k \in \mathbb{Z}$, is an isometry between $E_{\omega}^{2}$ and $\ell^{2}(\mathbb{Z})$. This map $S_{\omega}$ is an isomorphism between $E_{\omega}^{p}$ and $\ell^{p}(\mathbb{Z})$ when $h=\pi / \omega$ and $1<p<\infty$. This fails if $p=1$ or $p=\infty$.

The problem addressed in this paper is to extend the Shannon theorem to functions $f \in L^{p}\left(\mathbb{R}^{n}\right), n>1,1<p<\infty$. We are given an integrable compact set $K \subset \mathbb{R}^{n}$ and we want to know if every $f \in L^{p}\left(\mathbb{R}^{n}\right)$ whose Fourier transform is supported by $K$ can be recovered from its samples on the grid $\Gamma=\mathbb{Z}^{n}$. The general case where $\mathbb{Z}^{n}$ is replaced by an arbitrary lattice $\Gamma$ will follow by a linear change of variables.

\section{Stable SAMPling AND STABle interpolation}

Let $K \subset \mathbb{R}^{n}$ be a compact set and $p \in(1, \infty)$. Let $E_{K}^{p}$ be the closed subspace of $L^{p}\left(\mathbb{R}^{n}\right)$ consisting of all $f \in L^{p}\left(\mathbb{R}^{n}\right)$ whose Fourier transform $\hat{f}$ is supported by $K$.

$$
E_{K}^{p}=\left\{f \in L^{p}\left(\mathbb{R}^{n}\right): \operatorname{supp} \hat{f} \subset K\right\}
$$

The Fourier transform $\hat{f}$ of $f$ is defined by

$$
\hat{f}(\xi)=\int_{\mathbb{R}^{n}} \exp (-i x \cdot \xi) f(x) d x, \xi \in \mathbb{R}^{n},
$$

Date: March 24, 2013.

The third author is supported by the Generalitat de Catalunya (grant 2009 SGR 1303) and the Spanish Ministerio de Economía y Competividad (project MTM201127932-C02-01). Part of this work was done while he was staying at the Center for Advance Studies in Oslo, and he would like to express his gratitude to the institute for the hospitality. 
where $x \cdot \xi=x_{1} \xi_{1}+\cdots+x_{n} \xi_{n}$. When $1 \leq p \leq 2$ the Fourier transform $\hat{f}$ belongs to $L^{q}$ with $1 / p+1 / q=1$. If $p>2$ this Fourier transform is defined in the distributional sense. The following lemmata are well known.

Lemma 2.1. If $K \subset \mathbb{R}^{n}$ is a compact set and $1 \leq p \leq \infty$ the restriction operator $S^{p}$ which is defined by

$$
f \mapsto(f(k))_{k \in \mathbb{Z}^{n}}
$$

is continuous form $E_{K}^{p}$ to $\ell^{p}\left(\mathbb{Z}^{n}\right)$.

The restriction of $f$ to any uniformly separated sequence belongs to $\ell^{p}$. This the well-known Plancherel-Polya inequality.

Lemma 2.2 (Poisson formula). If $F$ is a compactly supported distribution and if $G(x)=\sum_{k \in \mathbb{Z}^{n}} F(x-2 \pi k)$ then the Fourier coefficients $c(k), k \in \mathbb{Z}^{n}$, of $G$ and the Fourier transform $\hat{F}$ of $F$ are related by

$$
c(k)=(2 \pi)^{-n} \hat{F}(k), k \in \mathbb{Z}^{n} .
$$

If $1<p<\infty$ we denote by $\mathcal{F} L^{p}$ the Banach space of all Fourier transforms of functions in $L^{p}\left(\mathbb{R}^{n}\right)$. The norm of $\hat{f}$ in $\mathcal{F} L^{p}$ is the norm of $f$ in $L^{p}$. The space of restrictions of $\mathcal{F} L^{p}$ to an open set $\Omega$ will be denoted by $\mathcal{F} L^{p}(\Omega)$. Similarly $\mathcal{F} \ell^{p}$ will denote the Banach space consisting of all $2 \pi \mathbb{Z}^{n}$ periodic functions (or distributions) whose Fourier coefficients belong to $\ell^{p}\left(\mathbb{Z}^{n}\right)$ and its norm is the $\ell^{p}$ norm of its coefficients.

Lemma 2.3. For $F \in \mathcal{F} \ell^{p}$ and for $\phi$ in the Schwartz class $\mathcal{S}$ the product $\phi F$ belongs to $\mathcal{F} L^{p}$

Indeed let $F(x)=\sum_{k \in \mathbb{Z}^{n}} c(k) \exp (i k \cdot x)$ where $c(k) \in \ell^{p}$ and let $\theta$ be the Fourier transform of $\phi$. Then the Fourier transform of $\phi F$ is $u(x)=\sum_{k \in \mathbb{Z}^{n}} c(k) \theta(x-k)$. By the Holder inequality

$$
|u(x)|^{p} \leq\left(\sum_{k \in \mathbb{Z}^{n}}|c(k)|^{p} \theta(x-k)\right)\left(\sum_{k} \theta(x-k)\right)^{p-1} .
$$

Thus it follows that $\|u\|_{p}^{p} \leq C \sum_{k \in \mathbb{Z}^{n}}|c(k)|^{p}$.

From now on the compact set $K$ is assumed to be regular.

Definition 2.1. A compact set $K \subset \mathbb{R}^{n}$ is regular if the following two conditions hold

(a) $K$ is connected

(b) for each $x_{0}$ belonging to the boundary $\Gamma=\partial K$ of $K$ there exist a neighborhood $V$ of $x_{0}$, a suitable coordinate system $\mathcal{R}_{x_{0}}$ and a continuous function $A_{x_{0}}: \mathbb{R}^{n-1} \mapsto \mathbb{R}$ such that $\Gamma$ coincides on $V$ with the graph of $A_{x_{0}}$ in $\mathcal{R}_{x_{0}}$ and $K$ coincides on $V$ with $\left\{x_{n} \geq A_{x_{0}}\left(x^{\prime}\right), x^{\prime}=\left(x_{1}, \ldots, x_{n-1}\right)\right\}$. 
A Lipschitz domain is regular. Definition 2.1 is required in this note since the counter example which is given below is not a Lipschitz domain.

We denote by $\mathcal{S}=\mathcal{S}\left(\mathbb{R}^{n}\right)$ the Schwartz class. We then have.

Lemma 2.4. If $1<p<\infty$ and if $K$ is regular, then $\mathcal{S} \cap E_{K}^{p}$ is dense in $E_{K}^{p}$.

Proof. Let $f \in E_{K}^{p}$. Then the compact support $L$ of $F=\hat{f}$ is contained in $K$. Using a smooth partition of the identity we split $F$ into a finite sum of pieces for which the local description of $K$ can be used. Then the interior $\Omega$ of $K$ is locally defined by $x_{n}>A\left(x_{1}, \ldots, x_{n-1}\right)$ while $K$ is defined by $x_{n} \geq A\left(x_{1}, \ldots, x_{n-1}\right)$ where $A$ is a continuous function. Let $F_{\varepsilon}=F\left(x_{1}, \ldots, x_{n-1}, x_{n}-\varepsilon\right)$ where $\varepsilon>0$ is a small positive number. The support $L_{\varepsilon}$ of $F_{\varepsilon}$ is $L_{\varepsilon}=L+\varepsilon e_{n}$ where $e_{n}=(0, \ldots, 0,1)$. Therefore $L_{\varepsilon}$ is contained in $\Omega$. Moreover $\left\|F_{\varepsilon}-F\right\|_{\mathcal{F} L^{p}}$ tends to 0 with $\varepsilon$. Indeed for every $f \in L^{p}$ Lebesgue dominated convergence theorem implies that $\left\|\left[\exp \left(i \varepsilon x_{n}\right)-1\right] f\right\|_{p} \rightarrow 0$ as $\varepsilon$ tends to 0 . To conclude the proof it suffices to approximate $F$ by a test function whose compact support is contained in $\Omega$. To reach this goal we replace $F_{\varepsilon}$ by the convolution product $F_{\varepsilon} \star \theta$ where $\theta$ is a smooth bump function supported by a sufficiently small ball $|x| \leq \eta$ with $\int \theta=1$.

Lemma 2.4 does not hold for a Riemann integrable compact set $K$. Here is a counter example in two dimensions. Let $K$ be the circle centered at 0 with radius 1 . The compact set $K$ is Riemann integrable. We consider the arc length measure $d \sigma$ on $K$ and its inverse Fourier transform $f$, that is a Bessel function that decays as $|x|^{-1 / 2}$ when $x \rightarrow$ $\infty$. Thus $f$ belongs to $E_{K}^{p}$ for $p>4$ but $f$ is not the limit in $L^{p}$ of a sequence of test functions in $E_{K}^{p}$ because there are no test functions in $E_{K}^{p}$. Any test function $g$ will belong to $L^{2}\left(\mathbb{R}^{2}\right)$ and $\hat{g}$ will be supported in $K$ that has measure 0 , thus it will vanish.

We now follow the seminal work of H. J. Landau [2].

Definition 2.2. A point set $\Gamma \subset \mathbb{R}^{n}$ is a set of stable sampling for $E_{K}^{p}$ if there exists a constant $C$ such that

$$
f \in E_{K}^{p} \Rightarrow\|f\|_{p}^{p} \leq C \sum_{\gamma \in \Gamma}|f(\gamma)|^{p}
$$

In other words $\Gamma \subset \mathbb{R}^{n}$ is a set of stable sampling for $E_{K}^{p}$ if $S^{p}$ : $E_{K}^{p} \mapsto \ell^{p}(\Gamma)$ is an isomorphism between $E_{K}^{p}$ and its image in $\ell^{p}(\Gamma)$. If this condition is not satisfied two problems may occur. The first one is named aliasing. Aliasing means that there exists a function $f \in E_{K}^{p}, f \neq 0$, such that $f(\gamma)=0, \gamma \in \Gamma$. Even if aliasing does not occur the reconstruction of $f$ from its samples $f(\gamma), \gamma \in \Gamma$, is not stable when (2.5) is not satisfied. Let $|E|$ denote the Lebesgue measure of a set $E$. 
When $\Gamma=\mathbb{Z}^{n}$, by Lemma 2.2 we can "code" the samples $f(\gamma)$ in a periodic function $G \in \mathcal{F}\left(\ell^{p}\right)$ as follows. Let $F$ be the Fourier transform of $f$. It has compact support and we can periodize it:

$$
G(x)=\sum_{k \in \mathbb{Z}^{n}} F(x-2 \pi k) .
$$

By Lemma 2.2, the Fourier coefficients $c(k)$ of $G$ satisfy $c(k)=f(-k)$. Hence, the sampling inequality (2.5), on the Fourier side, amounts to say that all $F \in \mathcal{F}\left(L^{p}\right)$ supported in $K$ are controlled by its periodized $G \in \mathcal{F}\left(\ell^{p}\right)$.

Lemma 2.5. Let $K \subset \mathbb{R}^{n}$ be a Riemann integrable compact set.

(a) If $\mathbb{Z}^{n}$ is a set of stable sampling for $E_{K}^{p}$ then

$$
\forall k \in \mathbb{Z}^{n}, k \neq 0 \Rightarrow|K \cap(K+2 \pi k)|=0 .
$$

(b) Conversely if the sets $K+2 \pi k, k \in \mathbb{Z}^{n}$, are pairwise disjoint, then $\mathbb{Z}^{n}$ is a set of stable sampling for $E_{K}^{p}$.

Observe that the sufficient condition (b) is more demanding than (a) and Lemma 2.5 does not fully answer the problem.

Proof. If (2.6) is not satisfied there exists a $k_{0} \in \mathbb{Z}^{n}$ such that the Riemann integrable set $K \cap\left(K-2 \pi k_{0}\right)$ has a positive measure. Therefore this set contains a small ball $B$. Then $B \subset K$ and $B+2 \pi k_{0} \subset K$. Let $f$ be any test function whose Fourier transform is supported by $B$. Then $g(x)=\left(\exp \left(2 \pi i k_{0} \cdot x\right)-1\right) f(x)$ belongs to $E_{K}^{p}$ and vanishes on $\mathbb{Z}^{n}$. Aliasing occurs.

We now prove (b). Let $F$ be the Fourier transform of the function $f \in E_{K}^{p}$ and $G$ its periodized version as above. If $\varepsilon>0$ is small enough and if $B(0, \varepsilon)$ is the ball centered at 0 with radius $\varepsilon$, the compact set $K^{\prime}=K+B(0, \varepsilon)$ still satisfies (b). Let $\phi$ be a test function supported by $K^{\prime}$ and such that $\phi(x)=1$ on $K$. This implies $F=\phi G$ and we can apply Lemma 2.3 .

Here is an example illustrating Lemma 2.5. Let us assume that $K$ is the disc $|x| \leq r$. If $0<r<\pi$ condition (b) is satisfied and if $r>\pi$ condition (a) does not hold. However Lemma 2.5 does not give any answer if $r=\pi$. To treat this case, we consider the cube $Q=[-\pi, \pi]^{n}$ to which Theorem 3.1 below can be applied. Therefore $\mathbb{Z}^{n}$ is a set of stable sampling for $E_{Q}^{p}$. Since $K \subset Q, \mathbb{Z}^{n}$ is a set of stable sampling for $E_{K}^{p}$.

When $p=2$, stable sampling is equivalent to (2.6). The goal of this note is to show that this property does not suffice when $p \neq 2$.

Definition 2.3. We say that $\Gamma \subset \mathbb{R}^{n}$ is a set of stable interpolation if every sequence $a(\gamma) \in \ell^{p}(\Gamma)$ can be interpolated by a function $f$ in $E_{K}^{p}$.

It means that there exists $f \in E_{K}^{p}$ such that $f(\gamma)=a(\gamma), \gamma \in \Gamma$. 
Lemma 2.6. Let $K \subset \mathbb{R}^{n}$ be a Riemann integrable compact set.

(a) If $\mathbb{Z}^{n}$ is a set of stable interpolation for $E_{K}^{p}$ then

$$
\bigcup_{k \in \mathbb{Z}^{n}}(K+2 \pi k)=\mathbb{R}^{n}
$$

(b) Conversely if $\Omega$ is the interior of $K$ and if

$$
\bigcup_{k \in \mathbb{Z}^{n}}(\Omega+2 \pi k)=\mathbb{R}^{n}
$$

then $\mathbb{Z}^{n}$ is a set of stable interpolation for $E_{K}^{p}$.

Here also the sufficient condition (b) is more demanding than (a). The proof is similar to the one used in Lemma 2.5. It is left to the reader.

We want to know when $S^{p}: E_{K}^{p} \rightarrow \ell^{p}\left(\mathbb{Z}^{n}\right)$ is an isomorphism. If it is the case, Lemma 2.5 and 2.6 imply that the translated sets $K+2 \pi k, k \in$ $\mathbb{Z}^{n}$, are a partition of $\mathbb{R}^{n}$ up to sets of measure 0 . We then say that $K$ is a fundamental domain for $\mathbb{Z}^{n}$. But the converse implication is not true. The fact that $K$ is a fundamental domain for $\mathbb{Z}^{n}$ does not imply that the operator $S^{p}$ is an isomorphism between $E_{K}^{p}$ and $\ell^{p}\left(\mathbb{Z}^{n}\right)$ when $p \neq 2$. This will be proved in the next section.

\section{Our Result}

A Borel function $m(x)$ is a multiplier of $\mathcal{F} L^{p}$ if we have

$$
F(x) \in \mathcal{F} L^{p} \Rightarrow m(x) F(x) \in \mathcal{F} L^{p}
$$

and if a constant $C$ exists such that

$$
\|m(x) F(x)\|_{\mathcal{F} L^{p}} \leq C\|F(x)\|_{\mathcal{F} L^{p}}
$$

This does not make any sense if $2<p$ since $F(x)$ may be a distribution. This issue is settled by the following remarks. It suffices to prove (3.2) when $f \in L^{2} \cap L^{p}$ and a density argument yields the general case. Moreover if $m$ is a multiplier of $\mathcal{F} L^{p}$ then $m$ is also a multiplier of $\mathcal{F} L^{q}$ when $1 / p+1 / q=1$. This reduces the case $p>2$ to $1<q<2$.

The following lemma will be seminal in the proof of Theorem 3.1.

Lemma 3.1. Let $m \in L^{\infty}\left(\mathbb{R}^{n}\right)$ be a compactly supported function. Then the following two properties are equivalent

(a) $m$ is a multiplier of $\mathcal{F} L^{p}$

(b) m maps $\mathcal{F} \ell^{p}$ into $\mathcal{F} L^{p}$

Proof. For proving (a) $\Rightarrow$ (b) let us denote by $\phi$ a smooth and compactly supported function such that $\phi=1$ on a neighborhood of the compact support of $m$. If $G \in \mathcal{F} \ell^{p}$ we have $\phi G \in \mathcal{F} L^{p}$ by Lemma 2.3 and $m G=m \phi G \in \mathcal{F} L^{p}$ since $m$ is a multiplier of $\mathcal{F} L^{p}$. We now prove (b) $\Rightarrow\left(\right.$ a). If $F \in \mathcal{F} L^{p}$ we have $m F=m \phi F$ as above. One uses a 
smooth partition of the identity to decompose $\phi F$ into a finite sum $F=\sum_{1}^{m} F_{j}$ where $F_{j} \in \mathcal{F} L^{p}$ and where the support of each $F_{j}$ is contained in a cube $Q_{j}$ centered at $x_{j}$ with side length 1 . It suffices to show that $m F_{j} \in \mathcal{F} L^{p}$ to conclude. We consider $G_{j}(x)=\sum_{k \in \mathbb{Z}^{n}} F_{j}(x-2 \pi k)$ and Lemmata 2.1 and 2.2 yield $G_{j} \in \mathcal{F} \ell^{p}$. Since $m$ maps $\mathcal{F} \ell^{p}$ into $\mathcal{F} L^{p}$ we have $m G_{j} \in \mathcal{F} L^{p}$. Let $\chi_{j}$ be a test function such that $\chi_{j}(x)=1$ on $Q_{j}$ and $\chi_{j}(x)=0$ on every $Q_{j}+2 k \pi, k \neq 0$. Then $m F_{j}=\chi_{j} m G_{j} \in \mathcal{F} L^{p}$.

Theorem 3.1. Let $K$ be a regular compact set. Assuming that $K$ is fundamental domain for $2 \pi \mathbb{Z}^{n}$ the following four properties are equivalent ones

(a) The indicator function of $K$ is a multiplier of $\mathcal{F} L^{p}$.

(b) The lattice $\mathbb{Z}^{n}$ is a set of stable sampling for $E_{K}^{p}$.

(c) The lattice $\mathbb{Z}^{n}$ is a set of stable interpolation for $E_{K}^{p}$.

(d) The operator $S^{p}: E_{K}^{p} \mapsto \ell^{p}\left(\mathbb{Z}^{n}\right)$ is an isomorphism.

Proof. Let us show that (a) implies (b). For $f \in E_{k}^{p}$ we consider $F$ and $G$ as above. If $K$ is fundamental domain for $\mathbb{Z}^{n}$, then formally $F=\chi_{K} G$. A priori $G$ could be a distribution if $p>2$, so we need to assume that $f \in \mathcal{S} \cap E_{k}$ which we may by Lemma 2.4. The property of stable sampling is first proved if $f \in \mathcal{S} \cap E_{K}^{p}$ and extended by continuity to the general case. Hence the sampling inequality (2.5) amounts to

$$
\left\|\chi_{K} G\right\|_{\mathcal{F} L^{p}} \leq\|G\|_{\mathcal{F} \ell^{p}}, \quad f \in E_{K}^{p} .
$$

This immediately shows, by Lemma 3.1 that (a) implies (b). The converse is also true because we can start from $G \in \mathcal{F}\left(\ell^{p}\right)$ and take $F=\chi_{K} G$ and $f$ the function that has $F$ as its Fourier transform. The assumption (b) means

$$
\|F\|_{\mathcal{F} L^{p}} \leq C\|f(k)\|_{\ell^{p}}
$$

which is the same as

$$
\|F\|_{\mathcal{F} L^{p}} \leq C\|G\|_{\mathcal{F} \ell^{p}} .
$$

The equivalence between (a) and (c) follows just by looking to the situation in terms of the sequence of Fourier coefficients of $G$, which is an arbitrary sequence in $\ell^{p}$.

The equivalence between (a) and (d) obviously follows from the preceding steps.

\section{An example}

4.1. Fefferman's theorem and stable sampling. Fefferman's theorem says that in any dimension $n \geq 2$ the indicator function $\chi_{B}$ of a ball $B$ is not a multiplier of $\mathcal{F} L^{p}$ when $p \neq 2$. Moreover the proof of Fefferman's theorem or an elementary reasoning shows that the result is local. If $\phi$ is any function which does not vanish identically on $\partial B$ 
then $\phi(x) \chi_{B}(x)$ is not a multiplier of $\mathcal{F} L^{p}$ if $p \neq 2$. This paves the way to our example.

The compact set $K \subset \mathbb{R}^{2}$ is defined as follows. We start with the square $Q=\left\{0 \leq x_{1}, x_{2} \leq 2 \pi\right\}$ and we call $D_{1}$ (resp. $D_{2}$ ) the closed discs centered at $x_{1}=(\pi, 0)$ (resp. $x_{2}=(\pi, 2 \pi)$ ) with radius $\pi$. Let $K_{1}=Q \cup D_{1}$ and $K=K_{1} \backslash D_{2}$. It is trivial to prove that $K$ is a fundamental domain for $2 \pi \mathbb{Z}^{2}$ as in the picture:

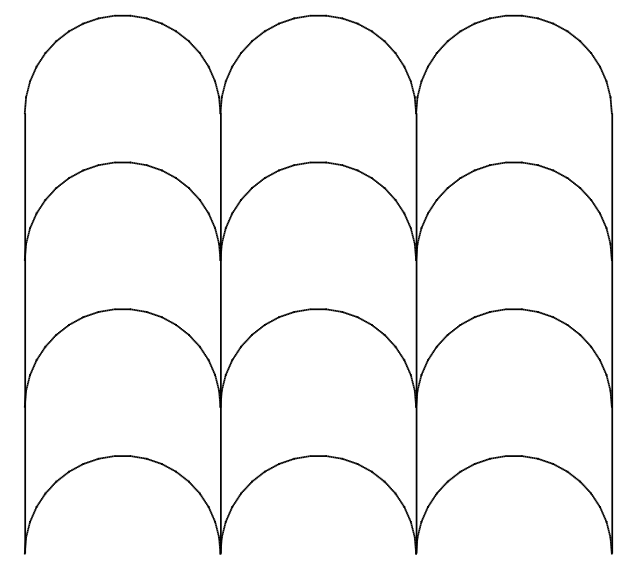

But we know from Fefferman's theorem that the indicator function of $K$ is not a Fourier multiplier of $\mathcal{F}\left(L^{p}\right)$ if $p \neq 2$. Therefore the functions $f \in E_{K}^{p}$ cannot be sampled on $\mathbb{Z}^{2}$ if $p \neq 2$.

\section{IRREgular SEQUENCES AND MULTIPLIERS}

A sequence $\Lambda \subset \mathbb{R}^{n}$ is a complete interpolating sequence for $E_{K}^{p}$ when for any sequence of values $v_{\lambda} \in \ell^{p}(\Lambda)$ there is a unique function $f \in E_{K}^{p}$ such that $f(\lambda)=v_{\lambda}$. It can be seen that complete interpolating sequences are simultaneously stable sampling and stable interpolating sequences.

We are not assuming now that $\Lambda$ has any structure. In such generality very little is known. In the case of dimension one, and $K$ being an interval there is a complete description of the complete interpolating sequences in $E_{I}^{p}$ due to Lyubarskii and Seip [3] that generalizes to any $p>1$ the description of Pavlov, [4] when $p=2$.

Nevertheless it is still possible to see that the existence of complete interpolating sequences is related to the boundedness of the multiplier at least in one direction.

Theorem 5.1. Given any compact $K$, and a complete interpolating sequence $\Lambda$ for the space $E_{K}^{p}$, the function $\chi_{K}$ is a multiplier for $\mathcal{F} L^{p}$

Proof. Take a smooth compactly supported function $\phi$ such that $\phi \equiv 1$ in a neighborhood of $K$. If $\widehat{F}=\phi$, then for any $f \in E_{K}^{p}$ we have the 
reproducing formula:

$$
f(z)=f \star F(z)=\int_{\mathbb{R}^{n}} f(x) F(z-x) d x .
$$

Consider the following two operators:

$$
\begin{array}{r}
T_{1}: L^{p}\left(\mathbb{R}^{n}\right) \rightarrow \ell^{p}(\Lambda) \\
f \rightarrow\{f \star F(\lambda)\}_{\lambda \in \Lambda}
\end{array}
$$

Clearly the operator $T_{1}$ is linear and bounded by the PlancherelPolya inequality since the sequence $\Lambda$ is separated and $f \star F \in E_{B}^{p}$, where $B$ is a ball containing the support of $\phi$.

Now consider the operator:

$$
T_{2}: \ell^{p}(\Lambda) \rightarrow E_{K}^{p}
$$

that to any sequence of values $\left\{v_{\lambda}\right\} \in \ell^{p}(\Lambda)$ associates the unique function $f \in E_{K}^{p}$ such that $f(\lambda)=v_{\lambda}$. This function exists because we assume that $\Lambda$ is a complete interpolating sequence. Moreover it defines a bounded linear operator. Thus the composition operator $T=$ $T_{2} \circ T_{1}$ maps $L^{p}\left(\mathbb{R}^{n}\right)$ to $E_{K}^{p}$ linearly and it is bounded. Because of the reproducing property, and the fact that $\Lambda$ is a uniqueness set for $E_{K}^{p}$ it follows that $T$ is a projection, i.e. $T \circ T=T$.

We are going to produce now another bounded projection invariant under translations. Denote by $\tau_{x}$ the translation operator by $x$. Then $T_{x}=\tau_{-x} T \tau_{x}$ is another projection with the same norm as $T$. We average them over a big ball and denote

$$
T_{R}=\frac{1}{|B(0, R)|} \int_{B(0, R)} T_{x} d x .
$$

The operator $T_{R}$ is again a projection with norm bounded by the norm of $T$. Since all the operators $T_{R}: L^{p} \rightarrow L^{p}$ are bounded uniformly, by the Banach-Alouglou theorem we can extract a sequence sequence $R_{n} \rightarrow \infty$ such that $T_{R_{n}}$ converges to a bounded operator $\widetilde{T}$ in the weak operator topology, i.e $\left\langle f, T_{R_{n}}(g)\right\rangle \rightarrow\langle f, \widetilde{T}(g)\rangle$ for all $f \in L^{q}$ and $g \in L^{p}$. The linear operator $\widetilde{T}$ is a projection and has bounded norm (all these properties are inherited from the $T_{R_{n}}$ ). We will see now that it commutes with the translations. Indeed if we fix $y \in \mathbb{R}^{n}$,

$$
\begin{aligned}
\tau_{y} T_{R} & =\frac{1}{|B(0, R)|} \int_{B(0, R)} \tau_{y-x} T \tau_{x} d x= \\
& =\frac{1}{|B(0, R)|} \int_{B(y, R)} \tau_{-x} T \tau_{x+y} d x= \\
& =\frac{1}{|B(0, R)|} \int_{B(0, R)} \tau_{-x} T \tau_{x+y} d x+G_{R},
\end{aligned}
$$

where $\left\|G_{R}\right\|=O(1 / R)$. 
Thus $\left\|T_{R_{n}} \tau-\tau T_{R_{n}}\right\| \rightarrow 0$ for any translate $\tau$, but since $T_{R_{n}} \tau-$ $\tau T_{R_{n}} \rightarrow \widetilde{T} \tau-\tau \widetilde{T}$ in the weak operator topology, then $\widetilde{T} \tau=\tau \widetilde{T}$.

Since $\widetilde{T}$ is a bounded linear operator that commutes with the translations then it is a convolution operator, i.e. it is given by a Fourier multiplier against a bounded function.

The fact that it is a projection onto the functions with spectra lying in $K$ implies that $\widetilde{T}$ is the multiplier given by $\chi_{K}$.

Corollary 5.1. For any smooth compact $K \subset \mathbb{R}^{n}$ there are no complete interpolating sequences for the space $E_{K}^{p}$ for any $p \neq 2,1<p<\infty$.

Proof. If such $\Lambda$ existed, $\chi_{K}$ will be a multiplier in $\mathcal{F}\left(L^{p}\right)$ but Fefferman theorem states that it cannot be bounded because $K$ has points with positive curvature.

\section{REFERENCES}

[1] C. Fefferman, The multiplier problem for the ball. A nn. of Math. (2) 94 (1971), $330-336$.

[2] H.J. Landau, Necessary density conditions for sampling and interpolation of certain entire functions. Acta Math. 117 (1967) 37-52.

[3] Y. Lyubarskii and K. Seip, Complete interpolating sequences for Paley-Wiener spaces and Muckenhoupt's $\left(A_{p}\right)$ condition. Rev. Mat. Iberoamericana 13 (1997) 361-376.

[4] B.S. Pavlov, The basis property of an exponential system and Muckenhoupt's condition, Dokl. Akad. Nauk SSSR 247 (1979) 37-40; english transl. in Sov. Math. Dokl. 20 (1979)

LAGA, Université Paris Nord, 99, avenue Jean-Baptiste Clément, 93430 Villetaneuse, France

CMLA, EnS-Cachan, 61, Avenue du Président-Wilson, 94235 Cachan CEDEX, France

Dpt. MAiA, Universitat de Barcelona, Gran Via 585, 08007 BarceLONA, SPAIN

E-mail address: matei@math.univ-paris13.fr, yves.meyer@cmla.ens-cachan.fr, jortega@ub.edu 